\title{
Relating perturbation magnitude to temporal gene expression in biological systems
}

\author{
Stephen J Callister ${ }^{\dagger 3}$, J Jacob Parnell ${ }^{\dagger 4,5}$, Michael E Pfrender ${ }^{4}$ and \\ Syed A Hashsham*1,2
}

\begin{abstract}
Address: ${ }^{1}$ Department of Civil and Environmental Engineering, Michigan State University, East Lansing, MI, 48824, USA, ${ }^{2}$ Center for Microbial Ecology, Michigan State University, East Lansing, MI, 48824, USA, ${ }^{3}$ Biological Separations and Mass Spectrometry, Pacific Northwest National Laboratory, Richland, WA 99352, USA, ${ }^{4}$ Department of Biology and Ecology Center, Utah State University, Logan, UT, 84322, USA and ${ }^{5}$ Center for Integrated BioSystems, Utah State University, Logan, UT, 84322, USA
\end{abstract}

Email: Stephen J Callister - stephen.callister@pnl.gov; J Jacob Parnell - jparnell@biology.usu.edu; Michael E Pfrender - pfrender@biology.usu.edu; Syed A Hashsham* - hashsham@egr.msu.edu

* Corresponding author †Equal contributors

Published: 19 March 2009

BMC Research Notes 2009, 2:43 doi:10.1 186/1756-0500-2-43

This article is available from: http://www.biomedcentral.com/I756-0500/2/43

(C) 2009 Hashsham et al; licensee BioMed Central Ltd.

This is an Open Access article distributed under the terms of the Creative Commons Attribution License (http://creativecommons.org/licenses/by/2.0), which permits unrestricted use, distribution, and reproduction in any medium, provided the original work is properly cited.
Received: 29 January 2009

Accepted: 19 March 2009

\begin{abstract}
Background: Most transcriptional activity is a result of environmental variability. This cause (environment) and effect (gene expression) relationship is essential to survival in any changing environment. The specific relationship between environmental perturbation and gene expression - and stability of the response - has yet to be measured in detail. We describe a method to quantitatively relate perturbation magnitude to response at the level of gene expression. We test our method using Saccharomyces cerevisiae as a model organism and osmotic stress as an environmental stress.
\end{abstract}

Results: Patterns of gene expression were measured in response to increasing sodium chloride concentrations $(0,0.5,0.7, \mathrm{I} .0$, and I.2 M) for sixty genes impacted by osmotic shock. Expression of these genes was quantified over five time points using reverse transcriptase real-time polymerase chain reaction. Magnitudes of cumulative response for specific pathways, and the set of all genes, were obtained by combining the temporal response envelopes for genes exhibiting significant changes in expression with time. A linear relationship between perturbation magnitude and response was observed for the range of concentrations studied.

Conclusion: This study develops a quantitative approach to describe the stability of gene response and pathways to environmental perturbation and illustrates the utility of this approach. The approach should be applicable to quantitatively evaluate the response of organisms via the magnitude of response and stability of the transcriptome to environmental change.

\section{Background}

The cause and effect relationship between perturbation and response is routinely used to study and characterize ecosystems in terms of stability. To extend this concept, we investigated the relationship between the affect of dif- ferent perturbation magnitudes on the dynamic level of transcriptional response. The establishment of a quantitative relationship between stress and response has implications for predictive capabilities related to the behavior of organisms in natural and engineered systems and can be 
established on many levels including community, population, proteome, and transcriptome.

Transcription is often compared by measuring the foldchange in relative expression $[1,2]$; however, a simple fold-change approach only accounts for one aspect of the response. Adjusting to perturbation spans a continuum of short-term stress responses, long-term acclimation, and genetic adaptation. Improved analytical tools for understanding the transcriptional mechanisms underlying variation in environmental tolerance will help address key issues such as what distinguishes generalists from specialists, and how do organisms increase tolerance $[3,4]$.

We evaluate a standard area of displacement to determine the quantitative relationship between perturbation and transcriptional response. We selected Saccharomyces cerevisiae as a model organism because of its existing knowledgebase related to gene expression, particularly in high salt concentration (model stress). Our method demonstrates a linear relationship resulting in a proportional response at very high perturbation magnitudes.

\section{Methods \\ Cell culture}

Saccharomyces cerevisiae W303 (ATCC 200060) was grown in batch culture at $30^{\circ} \mathrm{C}$ in YDP medium containing $2 \%$ bacto-peptone, $1 \%$ yeast extract, and $2 \%$ glucose. Cells were harvested by centrifugation, washed, and suspended in YDP to a final $O_{600}$ of approximately $0.16 .5 \mathrm{M} \mathrm{NaCl}$ was added in triplicate to early log phase $\left(\mathrm{OD}_{600}\right.$ of $\left.\sim 1.0\right)$ cells resulting in a final $\mathrm{NaCl}$ concentration of $0.5 \mathrm{M}, 0.7$ $\mathrm{M}, 1.0 \mathrm{M}$, or $1.2 \mathrm{M}$. Prior to salt addition, a $15 \mathrm{ml}$ sample was collected to characterize the base-line gene expression level. During perturbation, cells were harvested at regular intervals [5] until growth resumed as indicated by three consecutive increases in $\mathrm{OD}_{600}$.

\section{RNA extraction and purification}

Total RNA was extracted using the modified hot acid phenol extraction method [6]. A $5 \mu$ l sub-sample was removed and reserved for RNA quantification and to check for genomic DNA contamination. All RNA extractions were performed in a dedicated hood, cleaned to reduce or destroy residual RNase activity using either a 10\% bleach solution or RNase wipes (Ambion, Austin TX).

Extracted RNA was evaluated for DNA contamination using a no-RT control as described previously [7] with the following PCR conditions as follows: $94^{\circ} \mathrm{C} 2 \mathrm{~min}, 40$ cycles of $94^{\circ} \mathrm{C} 0.5 \mathrm{~min}, 55^{\circ} \mathrm{C} 0.5 \mathrm{~min}, 72^{\circ} \mathrm{C} 1 \mathrm{~min}$ followed by a final elongation step $72^{\circ} \mathrm{C} 1 \mathrm{~min}$. Amplified PCR products were detected on a 3\% agarose gel. Samples containing DNA contamination digested with Turbo ${ }^{\text {тм }}$
DNase (Ambion, Austin, TX) at $37^{\circ} \mathrm{C}$ for 30 minutes and reevaluated for DNA contamination.

\section{Gene selection and primer design}

Sixty genes were selected for observation during osmotic shock based on whether: a) the genes exhibit significant expression to osmotic shock $[5,8,9]$, b) the genes show connection to osmotic shock, although insignificant expression in microarray results [5], and c) the genes are connected with one of the three biochemical pathways associated with salt stress, namely gycolysis, glycerolipid, and the high-osmotic glycerol (HOG) pathway, based on the Kyoto Encyclopedia of Genes and Genomes (KEGG) database [10] (Table 1).

Primers for each gene were designed using Primer Express $^{\mathrm{TM}} 2.0$ (Perkin Elmer Applied Biosystems, Foster City, CA). Design parameters included a $\mathrm{T}_{\mathrm{m}}$ from $58^{\circ} \mathrm{C}$ to $62^{\circ} \mathrm{C}$, GC content from $45 \%$ to $55 \%$, length from $20 \mathrm{bp}$ to $22 \mathrm{bp}$, and amplicon length from $100 \mathrm{bp}$ to $110 \mathrm{bp}$. Additional design parameters were set according to the manufacturer's suggestions. Specificity of primers was evaluated by gel electrophoresis of the amplified products, and analyzing dissociation curves for amplicons following quantitative real-time PCR.

\section{Quantitative real-time PCR}

RNA samples were reverse transcribed using a High Capacity cDNA Conversion $\mathrm{Kit}^{\mathrm{TM}}$ (Applied Biosystems, Foster City, CA), purified using Qiagen PCR purification columns (Qiagen, Valencia, CA) and quantified at 260 nm. 10-20 ng of purified cDNA was added to a reaction cocktail containing the PCR buffer described earlier with $2.5 \%$ (vol/vol) SYBR ${ }^{\mathrm{TM}}$ Green I (Molecular Probes, Eugene, OR; 1000×), and $2.3 \mu \mathrm{M}$ 6-ROX (6-carboxy-x-rhodamine, Molecular Probes, Eugene, OR). PCR was performed on an ABI 7900 HT (Applied Biosystems, Foster City, CA) using the reaction conditions, minus the final elongation step, described earlier.

Calibration curves of template dilution series of a $101 \mathrm{bp}$ fragment of inorganic phosphatase and a 106 bp fragment of glycerol-3-phosphate were run in triplicate for each PCR run to normalize differences in amplicon sizes. The $101 \mathrm{bp}$ and $106 \mathrm{bp}$ fragments corresponded to the range of amplicons bounded by the primers designed for selected genes. PCR products were purified using 10,000 MWCO spin columns (Millipore, Bedford, MA), and quantified by absorbance at $260 \mathrm{~nm}$. Purified products were adjusted to the same absorbance and a 1:10 dilution series was prepared as the starting template for the standard curves. Four dilutions from each series $\left(10^{0}, 10^{-2}, 10^{-}\right.$ 4 , and $10^{-6}$ ) were evaluated for linearity and agreement in cycle threshold (Ct). 
Table I: Genes used in this study belonging to one of three osmotolerance biochemical pathways.

\begin{tabular}{|c|c|c|}
\hline Gene & Description & Pathway \\
\hline YAL038W & Pyruvate kinase & Glycolysis \\
\hline YBROI9C & UDP-glucose-4-epimerase & Glycolysis \\
\hline YBR029C & Phosphatidate cytidylyltransferase (CDP-diglyceride synthetase) & Glycerolipid \\
\hline YBRI96C & Glycolytic enzyme phosphoglucose isomerase & Glycolysis \\
\hline YCL004W & Phosphatidylglycerolphosphate synthase & Glycerolipid \\
\hline YCL040W & Glucokinase & Glycolysis \\
\hline YCROI2W & 3-phosphoglycerate kinase & Glycolysis \\
\hline YDL02IW & Homolog of Gpm Ip phosphoglycerate mutase & Glycolysis \\
\hline YDL022W & NAD-dependent glycerol-3-phosphate dehydrogenase & Glycerolipid \\
\hline YDL052C & I-acyl-sn-gylcerol-3-phosphate acyltransferase & Glycerolipid \\
\hline YDLI42C & Cardiolipin synthase & Glycerolipid \\
\hline YDLI68W & $\begin{array}{l}\text { Bifunctional enzyme containing both alcohol dehydrogenase and glutathione-dependent formaldehyde dehydrogenase } \\
\text { activities }\end{array}$ & Glycerolipid \\
\hline YDL235C & Phosphorelay intermediate protein & HOG \\
\hline YDL243C & Putative aryl-alcohol dehydrogenase & Glycerolipid \\
\hline YDR050C & Induced under stress conditions; triosephosphate isomerase & Gycolysis \\
\hline YDRI47W & Ethanolamine kinase & Glycerolipid \\
\hline YDR380W & Phenylpyruvate decarboxylase & Glycolysis \\
\hline YDR48IC & Repressible alkaline phosphatase & Glycerolipid \\
\hline YER026C & phosphatidylserine synthase & Glycerolipid \\
\hline YER062C & DL-glycerol-3-phosphatase & Glycerolipid \\
\hline YER073W & Mitochondrial aldehyde dehydrogenase & Glycerolipid \\
\hline YERII8C & Transmembrane osmosensor & HOG \\
\hline YERI78W & EI alpha subunit of the pyruvate dehydrogenase $(\mathrm{PDH})$ complex & Glycolysis \\
\hline YFL056C & Putative aryl-alcohol dehydrogenase & Glycerolipid \\
\hline YFR053C & Hexokinase isoenzyme I & Glycolysis \\
\hline YGL257C & Mannosyltransferase & Glycolysis \\
\hline YGR007W & Choline phosphate cytidylyltransferase & Glycerolipid \\
\hline YGR088W & Cytosolic catalase $\mathrm{T}$ & HOG \\
\hline YGRI70W & Phosphatidylserine decarboxylase & Glycerolipid \\
\hline YGR202C & Cholinephosphate cytidylyltransferase & Glycerolipid \\
\hline YGR240C & Alpha subunit of heterooctameric phosphofructokinase & Glycolysis \\
\hline YHL007C & Signal transducing kinase of the PAK ( $\mathrm{p} 2 \mathrm{I}$-activated kinase) family & HOG \\
\hline YHL032C & Glycerol kinase & Glycerolipid \\
\hline Gene & Description & Pathway \\
\hline YHRI23W & sn-I,2-diacylglycerol ethanolamine- and cholinephosphotranferase & Glycerolipid \\
\hline YILOI4W & Alpha-I,3-mannosyltransferase & Glycerolipid \\
\hline YILI47C & Histidine kinase osmosensor that regulates a MAP kinase cascade & HOG \\
\hline YILI55C & Mitochondrial glycerol-3-phosphate dehydrogenase & Glycerolipid \\
\hline YJLI28C & MAP kinase kinase & HOG \\
\hline YKL060C & Fructose I,6-bisphosphate aldolase & Glycolysis \\
\hline YKR03IC & Phospholipase D & Glycerolipid \\
\hline YLR006C & Cytoplasmic response regulator & HOG \\
\hline YLRII3W & Mitogen-activated protein kinase & HOG \\
\hline YLRI33W & choline kinase & Glycerolipid \\
\hline YLR362W & Signal transducing MEK kinase involved & HOG \\
\hline YLR377C & Fructose-I,6-bisphosphatase & Glycolysis \\
\hline YML004C & Monomeric glyoxalase I & HOG \\
\hline YML070W & Dihydroxyacetone kinase & Glycerolipid \\
\hline YMR037C & Transcriptional activator related to Msn4p & HOG \\
\hline YMR043W & Transcription factor & HOG \\
\hline YMRI05C & Phosphoglucomutase & Glycolysis \\
\hline YMRI69C & Aldehyde dehydrogenase that uses NAD+ as the preferred coenzyme & Glycolysis \\
\hline YMR303C & Glucose-repressible alcohol dehydrogenase II & Glycolysis \\
\hline YNLI30C & Cholinephosphotransferase & Glycerolipid \\
\hline YNR03IC & Suppressor of Sensor Kinase (SLNI) & HOG \\
\hline YOL056W & Homolog of Gpmlp phosphoglycerate mutase & Glycolysis \\
\hline YOL059W & NAD-dependent glycerol 3-phosphate dehydrogenase & Glycerolipid \\
\hline YORI20W & Putative NADP $(+)$ coupled glycerol dehydrogenase & Glycerolipid \\
\hline
\end{tabular}


Table I: Genes used in this study belonging to one of three osmotolerance biochemical pathways. (Continued)

\begin{tabular}{lll}
\hline YOR374W & Mitochondrial aldehyde dehydrogenase & Glycolysis \\
YPL017C & Putative S-adenosylmethionine-dependent methyltransferase & Glycolysis \\
YPL206C & Endoplasmic reticulum protein of unknown function & Gycerolipid \\
YPRII3W & Phosphatidylinositol synthase & Glycerolipid \\
\hline
\end{tabular}

\section{Algorithm}

To evaluate gene expression patterns over time to the applied perturbations, the relative response of an individual gene was calculated as:

$$
r(t)_{i}=\left(x_{i}(t)-x_{i}(0)\right) / x_{i}(0)
$$

where, $x_{i}(t)$ represents the arbitrary expression for gene $i$ at time $t$. Equation 1 represents the deviation of gene expression from the onset of the perturbation, $x_{i}(0)$. The aggregate relative response of all genes or pathways at a given time was calculated as the difference in the cumulative responses of genes making up the set or pathway:

$$
c(t)=\left(\sum_{1}^{i} x_{i}(t)-\sum_{1}^{i} x_{i}(0)\right) / \sum_{1}^{i} x_{i}(0) .
$$

This calculation for a given time period represents the relative response envelope of the aggregate. For the response of a gene to be included in the aggregate relative response, the gene must meet the following criteria: 1) a measured response in two of three replicates at each sampling event (five total), 2) the measured response was within the dynamic range of the standard curve used to determine its expression value, and 3) the fractional coefficient of variation among replicate measurements was less than 1 . This latter criterion was included because the cumulative response is highly subject to uncertainty from propagation of random error. To quantify the total amount of displacement, the area inscribed by the response envelope was determined by curve fitting using a polynomial regression model:

$$
\gamma(t)=\beta_{0}+\beta_{1} t_{1}+\beta_{2} t_{2}+\ldots \beta_{k} t_{k}
$$

Where, $\beta_{k}$ are the coefficients describing the shape of the relative response and $\gamma(t)$ is the specific model for each relative response envelope from the onset of the perturbation $(\mathrm{t}=0)$ to the last measurement.

\section{Results and discussion}

Cell growth in response to $\mathrm{NaCl}$ was typified by a lag phase followed by resumption of growth at a reduced rate (Fig. 1) [11-13]. The relative displacement of the selected genes indicates increased expression followed by decreased expression for all perturbation magnitudes tested (Fig. 2) including a return of some genes to pre-per- turbed levels (see Fig. 2A). While the lag phase has been hypothesized as an adjustment required for growth in new conditions [14], it remained unclear why progression through the cell cycle is arrested. By analyzing the response envelope to different salt concentrations, we found that the suspension of growth coincides with the reset to a new steady state (Figs 1 \&2). The increasing lag phase and decreased growth rates at greater salt concentrations suggest that exceeding 1.2 $\mathrm{M} \mathrm{NaCl}$ would result in failure to resume growth in agreement with other reported values [15-17]. Note, that while high salt concentration results in osmotic stress, high $\mathrm{NaCl}$ concentrations can also induce sodium toxicity; however, these results are limited to known osmotolerance mechanisms.

One of the most dominant genes in all four perturbations is GPD1 (glycerol-3-phosphate dehydrogenase). However, DAK1 (dihydroxyacetone kinase), GLO1 (oxidore-

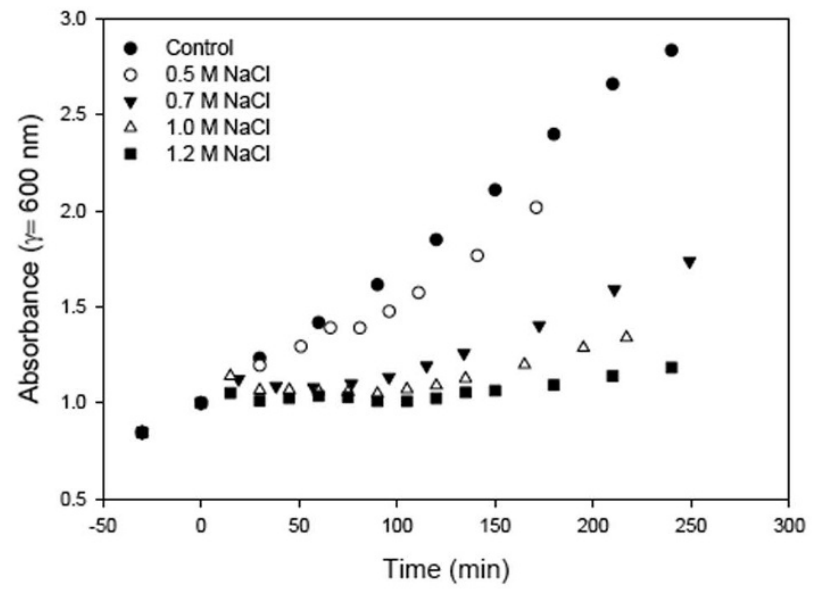

\section{Figure I}

Effect of elevated salt concentrations on the growth of S. cerevisiae. Growth in response to 5 press perturbations of $\mathrm{NaCl}$ addition to the growth medium resulting in the final $\mathrm{NaCl}$ concentrations indicated. S. cerevisiae displayed the typical behavior to $\mathrm{NaCl}$ addition, mainly an arrest in growth at or shortly after the onset of the perturbation followed by a period of adjustment, and then a resumption of growth at a lower rate. The lag period following addition of $\mathrm{NaCl}$ to a concentration of $0.5 \mathrm{M}$ was barely detectable, while at the I.2 $\mathrm{M} \mathrm{NaCl}$ perturbation resumption of growth was barely noticeable, which suggested at this perturbation magnitude the cell culture had been significantly impacted by the effects of osmotic shock and $\mathrm{NaCl}$ toxicity. 

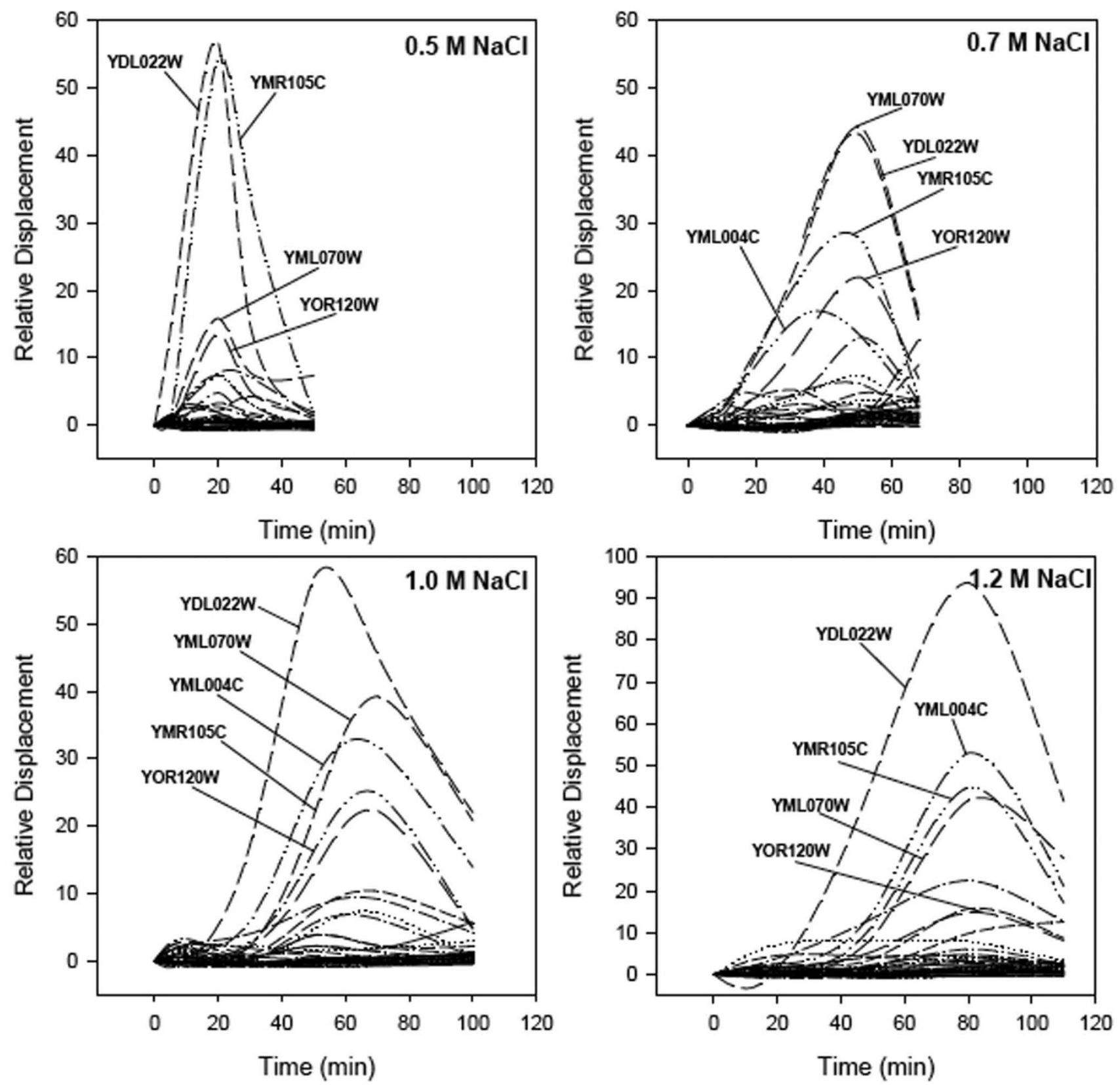

Figure 2

Relative response envelopes for salt-tolerance genes in S. cerevisiae determined by Q-RT-PCR following perturbation by addition of: A) $0.5 \mathrm{M} \mathrm{NaCl}$, B) $0.7 \mathrm{M} \mathrm{NaCl}$, C) I.0 M NaCl, and D) I.2 M NaCl. Three of the genes induced during salt stress (YDL022W, YML070W \& YORI20W) are responsible for glycerol production and correspond to the glycerolipid pathway. Two other genes with elevated expression, YML004C and YMRI05C, correspond to the highosmotic glycerol (HOG) and glycolysis pathways, respectively.

ductase), PGM2 (phosphoglucomutase), and GCY1, (phosphatidylserine synthase) follow similar patterns. These genes, in part, produce and regulate glycerol; essential for growth under osmotic stress [18-20]. GPD1, DAK1 and GCY1 are thought to be co-transcribed [19]. Glycerol is synthesized from glucose, and a rate-limiting enzyme in glycerol biosynthesis is the GPD1 gene product, which catalyzes reduction of dihydroxyacetone phosphate to glycerol 3-phosphate $[14,21,22]$. Although the halometric increase of GPD1 and DAK1 has been noted [23], the 

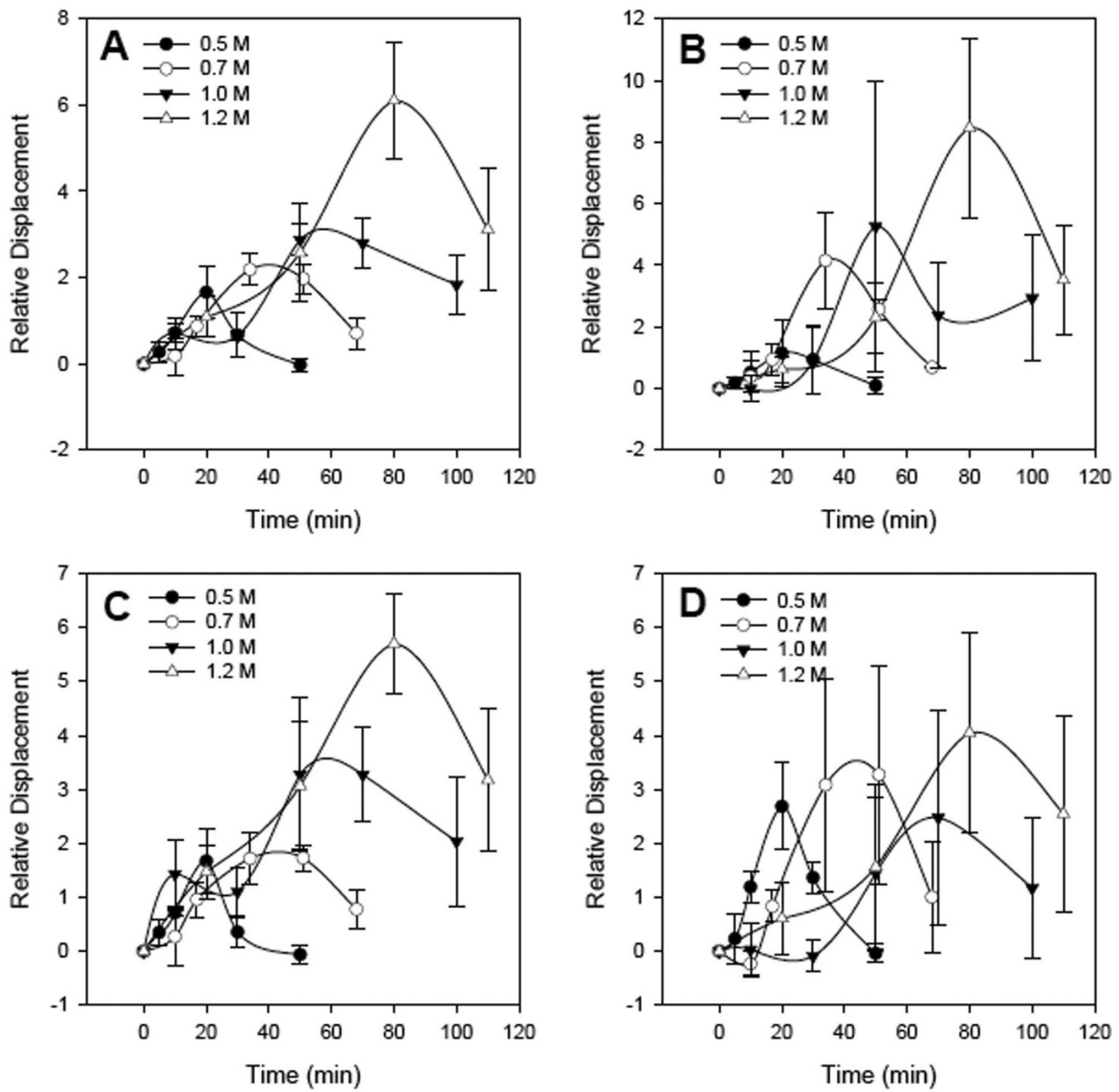

Figure 3

Relative response envelopes used to calculate strain for: A) the aggregate set of all genes, B) high-osmotic glycerol (HOG), C) glycerolipid metabolism, and D) glycolysis pathways. Relative displacement shown for the pathways was normalized by the number of genes making up each pathway. Comparison of response envelopes between pathways and the aggregate set of genes revealed different trends of behavior with increasing perturbation magnitude. These trends suggested a different contribution of each pathway to the aggregate relative response for each perturbation magnitude.

increase of GPD1 is proportional to the perturbation up to $1.2 \mathrm{M} \mathrm{NaCl}$, while the abundance of $D A K 1$ plateaus between 0.5 and $0.7 \mathrm{M} \mathrm{NaCl}$ (see Figs. 2C \&2D). GLO1 detoxifies a cytotoxic metabolite derived from dihydroxyacetone phosphate which increases during glycerol production [22]. PGM2 converts glucose-1 phosphate and glucose-6 phosphate [24] and is an important factor in glycolysis (eventually leading to glycerol production) as well as the production of other compatible solutes such as trehalose during salt stress.

The ecological parameters, resistance and resilience were estimated from the aggregate response envelops, the cumulative displacement of all measured genes (Fig. 3A). 


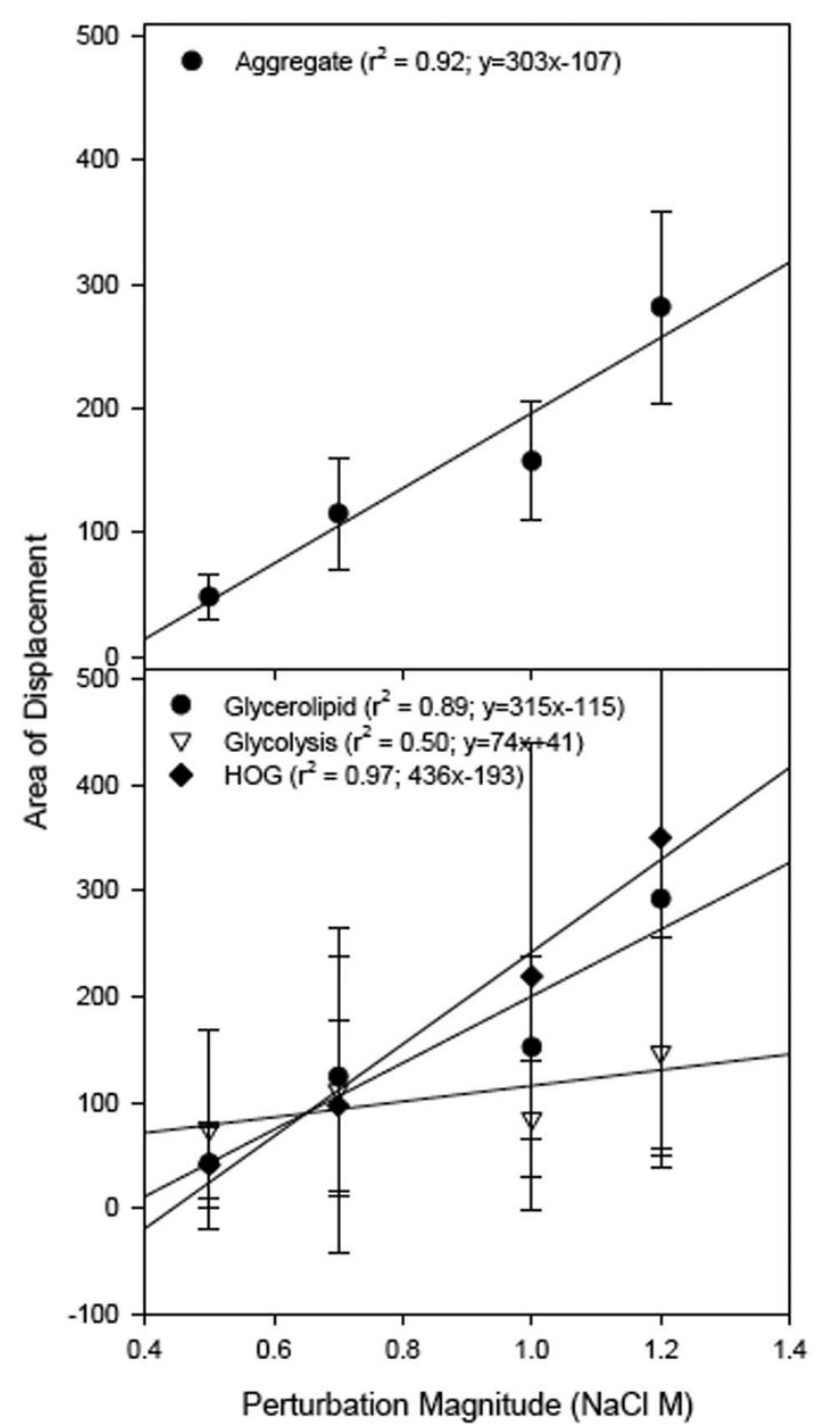

Figure 4

Relative area of displacement versus perturbation magnitude for the aggregate response of all genes (top), and genes grouped by biochemical pathway (bottom). A linear relationship was observed between stress and response, with the highest response resulting from the I.2 $\mathrm{M} \mathrm{NaCl}$ perturbation.

From the response envelopes, the degree of resistance was estimated as the maximum displacement, with a larger value for this displacement indicating a lesser degree of resistance [25]. Resilience, measured as the approximate duration of the response, was $\sim 1.4$-fold less for the $0.7 \mathrm{M}$ response compared to the $0.5 \mathrm{M}$ response and $\sim 3$-fold less for the $1.2 \mathrm{M}$ perturbation compared to the $0.5 \mathrm{M}$ perturbation. Response envelopes for the individual pathways involved in osmotic stress response, the high-osmotic glycerol (HOG) (Fig. 3B) and the Glycerolipid (Fig. 3C) synthesis exhibited monotonic behavior similar to the aggregate set of genes. Contrary to the monotonic increase in response envelope associated with increased stress seen in the two pathways mentioned above, the glycolysis pathway demonstrated a greater resilience at the $0.5 \mathrm{M}$ perturbation compared to the $1.2 \mathrm{M}$ perturbation (Fig. 3D).

The area of relative displacement increased in response to increased stress. For the aggregate of selected genes (Fig. $4 \mathrm{~A})$, the area of relative displacement associated with the $0.7 \mathrm{M}$ stress was approximately 2.4 fold greater with respect to the $0.5 \mathrm{M}$ stress. Overall, the area of displacement increased with stress and exhibited a high degree of linearity $\left(\mathrm{r}^{2}=0.92\right)$, suggesting a linear relationship between osmotic stress and transcriptional response. A linear trend in response to increased stress was also observed for the biochemical pathways (Fig. 4B) with the exception of the glycolysis pathway $\left(\mathrm{r}^{2}=0.50\right)$, where observed increase in the area of relative displacement in response to an increase in stress was not significant. The high degree of linearity for both the glycerolipid and HOG pathways is due to their direct association with osmotic stress (whereas glycolysis regulation is not solely influenced by osmotic stress). The proportional rate of increase (slope) was most dramatic for the HOG metabolism pathway and glycerolipid production. Because the area of relative displacement includes both the resistance and resilience components of stability, its larger rate of increase suggests these pathways are less stable compared to the glycolysis pathway.

The ability of a system, in this case the set of selected genes and pathways, to adjust to environmental changes has been described as resilience [26-29], and the ability to withstand the effects imposed by the changes - similar to the concept of buffering capacity - has been described as resistance $[25,28]$. Resilience at each perturbation magnitude $(0.5,0.7,1.0$, and $1.2 \mathrm{M} \mathrm{NaCl})$ was determined by the time required for either the aggregate set of genes or biochemical pathway to approach pre-perturbed displacement [25] or the new steady state of $S$. cerevisiae gene expression following exposure to high salt concentrations [23]. Resistance was estimated as the maximum displacement of the response envelope [25]. In terms of the aggregate set of genes and pathways, a larger stress resulted in decreased resilience (see Figs. 2 \&3) and less resistance.

\section{Conclusion}

Although our model determines the resilience and resistance of $S$. cerevisiae genes and pathways to elevated $\mathrm{NaCl}$ concentrations, this approach can be applied to measure the transcriptional response (positive or negative) to a range of environmental changes. This model is designed to quantify an overall response by determining the change 
in expression. In the terms of strain, or transcriptome response, down-regulated and up-regulated genes can be represented as a positive response value, for instance one gene that is up-regulated and another equally down-regulated will not cancel each other out, but the response value will be amplified.

Examining the response over a prolonged exposure to stress, rather than a snapshot approach, allows the quantification of the response to increased stress. This approach allows a direct comparison of the contribution of each gene or pathway on the aggregate transcriptome. The relationship between fitness and environmental conditions is inherently a multigenic phenomenon requiring this type of an approach. Understanding the relationship between stress, response, and resulting stability at the level of the transcriptome could provide predictive capabilities for other areas of biological research, such as engineering biostimulation for de-toxification of pollutants, and as a predictive indicator for adaptation to changing environments.

\section{Authors' contributions}

SJC and SAH designed this study, SJC collected and analyzed data, SJC, JJP and MEP interpreted data and wrote the manuscript, all authors read and approved the final draft.

\section{Acknowledgements}

This work was funded in part by the Center for Microbial Ecology and Intramural Research Grant Program at Michigan State University. Funding for this project was also provided by NSF grant DEB-02 I 2 2487 to MEP and a USU Center for Integrated BioSystems research grant.

We are thankful to Mr. Sean Spellman for help in sample collection. We are also thankful to Dr. Annette P. Thelen, Research Technology Support Facility at Michigan State University for help with sample preparation and quantitative real-time PCR

\section{References}

I. DeRisi JL, lyer VR, Brown PO: Exploring the metabolic and genetic control of gene expression on a genomic scale. Science 1997, 278:680-686.

2. Schena M, Shalon D, Davis RW, Brown PO: Quantitative monitoring of gene-expression patterns with a complementary DNA microarray. Science 1995, 270:467-470.

3. Bennett AF, Lenski RE: Experimental evolution and its role in evolutionary physiology. Amer Zool 1999, 39:346-362.

4. Kassen R, Rainey PB: The ecology and genetics of microbial diversity. Ann Rev Microbiol 2004, 58:207-23I.

5. Causton HC, Ren B, Koh SS, Harbison CT, Kanin E, Jennings EG, Lee TI, True HL, Lander ES, Young RA: Remodeling of yeast genome expression in response to environmental changes. Mol Biol Cell 200I, 12:323-337.

6. Schmitt ME, Brown TA, Trumpower BL: A rapid and simple method for preparation of RNA from Saccharomyces cerevisiae. Nucleic Acids Res 1990, 18:3091-3092.

7. Karsai A, Muller S, Platz S, Hauser MT: Evaluation of a homemade SYBR (R) Green I reaction mixture for real-time PCR quantification of gene expression. BioTechniques 2002, 32:790-796.

8. Gasch AP, Spellman PT, Kao CM, Carmel-Harel O, Eisen MB, Storz $G$, Botstein D, Brown PO: Genomic expression programs in the response of yeast cells to environmental changes. Mol Biol Cell 2000, I I:424I-4257.

9. Norbeck J, Pahlman AK, Akhtar N, Blomberg A, Adler L: Purification and characterization of two isoenzymes of DL-glycerol3-phosphatase from Saccharomyces cerevisiae - Identification of the corresponding GPPI and GPP2 genes and evidence for osmotic regulation of Gpp2p expression by the osmosensing mitogen-activated protein kinase signal transduction pathway. J Biol Chem 1996, 27 I:I3875-I388I.

10. Kanehisa M, Goto S: KEGG: Kyoto Encyclopedia of genes and genomes. Nucleic Acids Res 2000, 28:27-30.

II. Alexander MR, Tyers M, Perret M, Craig BM, Fang KS, Gustin MC: Regulation of cell cycle progression by Swe Ip and Hog Ip following hypertonic stress. Mol Biol Cell 200I, I 2:53-62.

12. Belli G, Gari E, Aldea M, Herrero E: Osmotic stress causes a G I cell cycle delay and downregulation of $\mathrm{Cln} 3 / \mathrm{Cdc} 28$ activity in Saccharomyces cerevisiae. Mol Microbiol 200I, 39:1022-1035.

13. Hohmann S, Mager WH: Yeast stress response. Chapman and Hall: New York, NY; 1997.

14. Mager $\mathrm{WH}$, Siderius $\mathrm{M}$ : Novel insights into the osmotic response of yeast. FEMS Yeast Res 2002, 2:25I-257.

15. Andreishcheva EN, Zvyagilskaya RA: Adaptation of yeasts to salt stress (Review). Prikl Biokhim Mikrobiol 1999, 35(3):243-256.

16. Blomberg A: Metabolic surprises in Saccharomyces cerevisiae during adaptation to saline conditions: questions, some answers and a model. FEMS Microbiol Lett 2000, 182: I-8.

17. Blomberg A: The osmotic hypersensitivity of the yeast Saccharomyces cerevisiae is strain and growth media dependent: Quantitative aspects of the phenomenon. Yeast 1997, 13:529-539.

18. Albertyn J, Hohmann S, Thevelein JM, Prior BA: GpdI, which encodes glycerol-3-phosphate dehydrogenase, is essential for growth under osmotic-stress in Saccharomyces cerevisiae, and its expression is regulated by the high-osmolarity glycerol response pathway. Mol Cell Biol 1994, I 4:4135-4I 44.

19. Nevoigt E, Stahl U: Reduced pyruvate decarboxylase and increased glycerol-3-phosphate dehydrogenase [NAD(+)] levels enhance glycerol production in Saccharomyces cerevisiae. Yeast 1996, I 2: | 33 |-1337.

20. Norbeck J, Blomberg A: Two-dimensional electrophoretic separation of yeast proteins using a non-linear wide range $(\mathrm{pH}$ 3-10) immobilized pH gradient in the first dimension; reproducibility and evidence for isoelectric focusing of alkaline (pl > 7) proteins. Yeast 1997, 13:1519-1534

21. Gancedo C, Gancedo JM, Sols A: Glycerol metabolism in yeasts. Eur J Biochem 1968, 5:165-172.

22. Inoue $Y$, Tsujimoto $Y$, Kimura A: Expression of the glyoxalase $I$ gene of Saccharomyces cerevisiae is regulated by high osmolarity glycerol mitogen-activated protein kinase pathway in osmotic stress response. / Biol Chem 1998, 273:2977-2983.

23. Rep M, Reiser V, Gartner U, Thevelein JM, Hohmann S, Ammerer G, Ruis H: Osmotic stress-induced gene expression in Saccharomyces cerevisiae requires Msn Ip and the novel nuclear factor Hot l p. Mol Cell Biol 1999, 19:5474-5485.

24. Hirata $Y$, Andoh T, Asahara T, Kikuchi A: Yeast glycogen synthase kinase-3 activates Msn2p-dependent transcription of stress responsive genes. Mol Biol Cell 2003, I 4:302-3 I2.

25. Hashsham SA, Fernandez AS, Dollhopf SL, Dazzo FB, Hickey RF, Tiedje JM, Criddle CS: Parallel processing of substrate correlates with greater functional stability in methanogenic bioreactor communities perturbed by glucose. Appl Environ Microbiol 2000, 66:4050-4057.

26. Deangelis DL: Energy flow, nutrient cycling, and ecosystem resilience. Ecology 1980, 61:764-77|.

27. Deangelis DL, Bartell SM, Brenkert AL: Effects of nutrient recycling and food-chain length on resilience. Am Nat 1989, 134:778-805.

28. Grimm V, Schmidt E, Wissel C: On the application of stability concepts in ecology. Ecol Model 1992, 63:|43-161.

29. Ives AR: Measuring resilience in stochastic systems. Ecol Monogr 1995, 65:217-233. 